\title{
Improvement of the Turajlić Method for the Estimation of Gaussian Noise in Images
}

\author{
Manuel G. Forero ${ }^{1(\bowtie)}\left(\mathbb{D}\right.$, Sergio L. Miranda ${ }^{1}$ (D), and Carlos Jacanamejoy-Jamioy ${ }^{2}$ (D) \\ 1 Semillero Lún, Facultad de Ingeniería, Universidad de Ibagué, Ibagué, Colombia \\ \{manuel. forero, sergio.miranda\} @unibague.edu.co \\ 2 Semillero Lún, Facultad de Ciencias Naturales y Matemáticas, \\ Universidad de Ibagué, Ibagué, Colombia \\ carlos.jacanamejoy@unibague.edu.co
}

\begin{abstract}
Gaussian noise estimation is an important step in some of the more recently developed noise removal methods. This is a difficult task and although several estimation techniques have been proposed recently, they generally do not produce good results. In a previous comparative study, among several noise estimation techniques, a method proposed in 2017 by Turajlić was found to give the best results. Although acceptable, they are still far from ideal. Therefore, several changes to this method are introduced in this paper to improve the estimation. Tests on monochromatic images contaminated with different levels of Gaussian noise showed that the modified method produces a significant improvement in the estimation of Gaussian noise, over $35 \%$, at a slightly higher computational cost.
\end{abstract}

Keywords: Noise estimation · Gaussian noise $\cdot$ Image filtering $\cdot$ Smoothing filters $\cdot$ Noise reduction

\section{Introduction}

A common problem in image processing is noise, which affects the further analysis of the information contained in the images. For this reason, different methods of noise removal have been developed and several of the latest image filtering and edge detection techniques require an estimation of the noise level to obtain better results, since in this way their parameters can be automatically adjusted to make the filtering more or less aggressive, or in the case of edge detectors, to consider as borders only those abrupt changes that are greater than those produced by the noise. These techniques include: phase congruency to detect edges [1], smoothing filters such as the anisotropic operator [2], and some considered state-of-the-art, based on sparse modelling such as K-SVD [3].

The most studied type of noise in the literature is Gaussian, since it is the most frequently found in digital images. In general, noise estimation techniques can be classified in two groups, depending on whether they are performed in the spatial or the frequency domain. Spatial techniques can be grouped into three, those that subdivide the image in blocks, those that employ filtering and the hybrids $[4,5]$ that have emerged in recent 
years. In general, block-based techniques [6] try to identify uniform areas where intensity variations are ideally due to noise alone, and then statistical tools are used to estimate noise. Those based on filters obtain a high pass or bandpass image from the original to separate the noise from the signal and then estimate the remaining noise level in the obtained image. Techniques where the estimation is done in the frequency domain [7] perform an analysis of the image spectrum using a decorrelation transformation, such as the discrete wave transformation.

In a recent study [8] it was found that estimation methods do not provide a good approximation in the vast majority of cases and, in general, hybrid methods provide a better estimate. Among these methods is a two-stage method developed by Turajlic [9]. In the first one, the image is filtered with a bandpass filter and the resulting image is divided into non-overlapping blocks of $64 \times 64$ pixels. The approximate dispersion of each block is then calculated using an equation that greatly reduces the cost of the computation compared to the standard deviation calculation. The second stage, in which these blocks are subdivided according to the minimum standard deviation obtained, is continued for $15 \%$ of the blocks. Finally, $85 \%$ of the sub-blocks with lower dispersion are used to obtain the noise level estimate.

This document is organized as follows. Section 2 describes the materials and experimental framework used to assess the performance of the proposed improvement. Section 3 describes the original Gaussian noise estimation algorithm from Turajlić. Section 4 introduces the proposed improvements to the original algorithm. Section 5 shows and discusses the results obtained. Finally, Sect. 6 presents our conclusions.

\section{Material}

Twenty $512 \times 512$ pixels monochrome images, two synthetic and eighteen taken from [10-12], were used to calculate the correction factor of the proposed method, shown in the Appendix. Forty $512 \times 512$ pixels monochrome images, two synthetics and thirty images taken from [10-12] were used for method validation and to compare the results against the original method. The images were contaminated with different levels of Gaussian noise from $\sigma=5$ to $\sigma=40$ in increments of 5 . The algorithms were written in Java as plugins of the freely available program ImageJ [13].

\section{Turajlić Method}

In the best case the noise estimate can be made easily, obtaining the standard deviation of a completely uniform region, ideally constituted by a single grey level, since it is assumed that the variations in intensity are due only to noise. Therefore, the most homogeneous blocks within the image are searched to make the estimation. However, in practice, it is not common to find completely uniform areas and signal variations affect noise estimation. To separate noise from signal, in some cases, band-pass or high-pass filters are used, as shown in Eq. 1, and the noise estimate is made on the obtained image. Following this trend, in 2017 Turajlić proposed a hybrid method for noise estimation [9], which consists of two parts. In the first one, the $15 \%$ of the most homogeneous areas of the image are found. To this end, the image is convoluted with the band-pass 
filter $h$ given in Eq. 1 and the resulting image is divided into non-overlapping blocks of $64 \times 64$ pixels. The dispersion of each block is obtained by Eq. 2, which greatly reduces the calculation time compared to the standard deviation method. Then, the minimum standard deviation $\sigma_{\min }$ of all blocks is found.

$$
\begin{gathered}
h=\begin{array}{ccc}
1 & -2 & 1 \\
-2 & 4 & -2 \\
1 & -2 & 1
\end{array} \\
\hat{\sigma}=\sqrt{\frac{\pi}{2}} \frac{1}{6(W-2)(H-2)} \sum_{\text {Image }}|I(x, y) * h|
\end{gathered}
$$

where $W$ and $H$ are the width and height of the block respectively.

In the second part, the homogeneous blocks found in the previous step, are subdivided into blocks of side equal to $\sigma_{\min } \times k$, where $k$ is a constant equal to 1.9 , found empirically. Then the dispersion of each sub-block is estimated using Eq. 2 and the $85 \%$ of the subblocks with less dispersion are defined as homogeneous regions. Finally, the noise level estimate is calculated as the average of the final homogeneous blocks.

\section{Enhanced Method}

As mentioned above, in the original method proposed by Turajlić as part of the first step, the $64 \times 64$ pixel block, which has the minimum dispersion $\sigma_{\min }$, is found. However, the dispersion of a block can be low, even if the noise in the image is high when the block is located in a very light or very dark region of the image, as the intensity cannot fall out of the available dynamic range [0, $L-1]$, where $L$ is usually 255 , which can lead to an erroneous estimation of the noise level, as can be observed in Fig. 1. To overcome this problem, it is verified that the gray level of all pixels within the block with the minimum dispersion $\sigma_{\min }$, is in the range given by the block mean $\mu \pm 3 \sigma_{\min }$, as shown in Eq. 3 . The block is discarded if the pixel intensities are not within the range. In this case, the next block with the lowest dispersion that meets the criteria given by Eq. 3 is searched for. Once found, its dispersion is taken as $\sigma_{\text {min }}$.
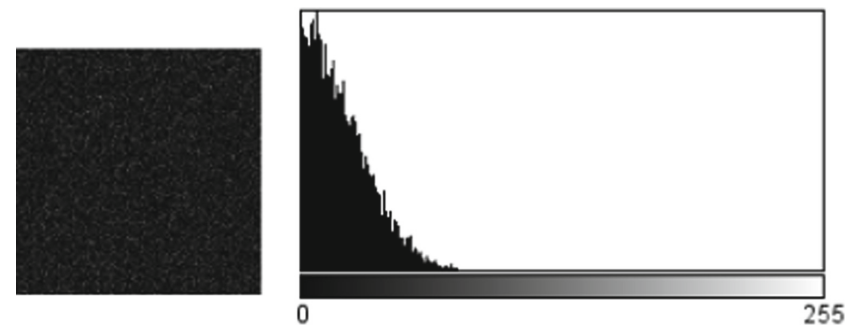

Fig. 1. Homogeneous dark area with an added noise of $\sigma=25$ and its histogram. As can be seen in the image histogram, the probability density function of the noise is incomplete, thus affecting the estimation. The estimate found for this case is $\sigma=15$.

$$
0 \leq \mu_{i, j}-3 \sigma_{\min } \wedge \mu_{i, j}+3 \sigma_{\min } \leq 255
$$


where $\mu_{i, j}$ the mean of pixel intensity of block.

$$
\hat{\sigma}=\sqrt{\frac{\pi}{2}} \frac{1}{A_{F}(W-2)(H-2)} \sum_{\text {Image }}|I(x, y) * h|
$$

Although Eq. 2, proposed by Turajlić, allows an acceptable approximation of noise in $64 \times 64$ pixel blocks, it was found experimentally not to work well for calculating noise in the smaller sub-blocks [8]. To correct this problem, the number 6 in the denominator of Eq. 2 is replaced in the second stage of the method by an $A_{F}$ fitting factor as shown in Eq. 4.

To find a suitable fitting factor $A_{F}$ a study was performed on 20 images, 2 synthetic and 18 natural ones taken from [10-12], which are shown in Fig. 2. These were contaminated with different levels of Gaussian noise from 5 to 40 in increments of 5. Two tests were performed per image for the same noise level where the value of $A_{F}$ varies from 4 to 10 in increments of 0.25 . Then, the setting value that gave the best noise estimation for each image was taken, taking into account that the final noise of each image is given by the natural noise plus the added noise. Therefore, the standard deviation of the noise in the final image, obtained from adding noise to each study image, was found by Eq. 5 [6], where $\sigma, \sigma_{0}[14]$ y $\sigma_{A D D}$ are the final, natural and added noise standard deviations. Finally, the function of the curve which best fits the data is calculated.

$$
\sigma=\sqrt{\sigma_{0}^{2}+\sigma_{A D D}^{2}}
$$

Figure 3 shows the set of fitting values obtained by each image, for each level of aggregated noise. To find the fitting value $A_{F}$, the function that best approximates the set of points was searched for. Two approaches were taken for this purpose. In the first approach, a third degree polynomial function was used and, in the second one, an exponential function was used, both shown in Fig. 3. The results obtained with

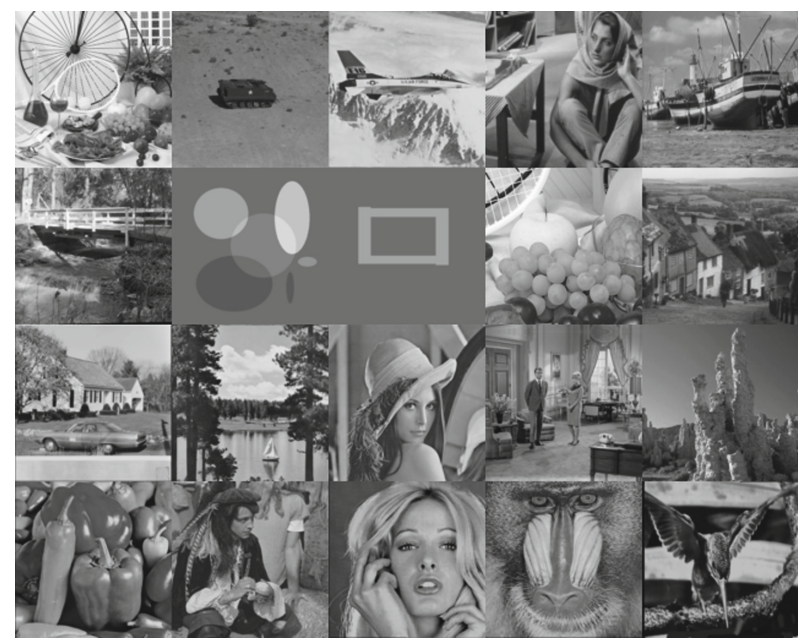

Fig. 2. Images employed to find $A_{F}$. 


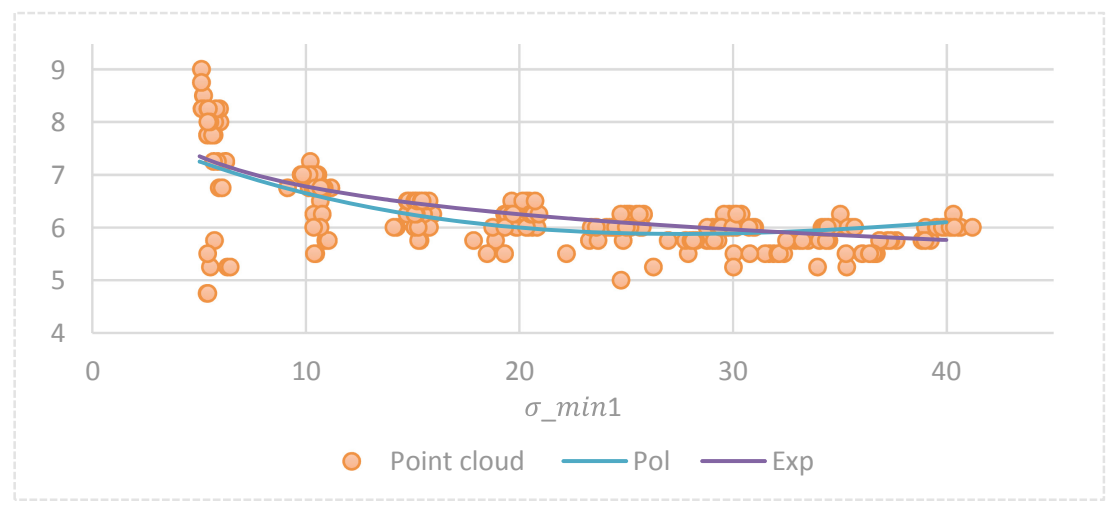

Fig. 3. $F_{A}$ point cloud and approximation functions.

both functions are denoted as Exp and Pol. In both approaches, the minimum standard deviation of the first stage $\sigma_{\min 1}$ was taken as the independent variable. The functions obtained are presented in Eqs. 6 and 7.

$$
\begin{gathered}
A_{f}=-0.00003779 \sigma_{\min 1}^{3}+0.005 \sigma_{\min 1}^{2}-0.189 \sigma_{\min 1}+8.09 \\
A_{F}=8.8912\left(\sigma_{\min 1}\right)^{-0.117}
\end{gathered}
$$

\section{Results}

Forty images contaminated with different levels of Gaussian noise were used to evaluate the behavior of the Turajlic method and the modified ones titled EXP and POL. It should be noted that these were evaluated on two percentages of area. $15 \%$ of the image, equivalent to 10 blocks, and with $11 \%$, equivalent to 7 blocks of $64 \times 64$ pixels. The execution time, mean square error (MSE) and the percentage of acceptable estimates were evaluated. This last measure is included, since in a previous work it was found that the published methods produce, in general, an estimate far from the real value. For a noise level estimate to be considered acceptable in this study, it must not exceed a 7\% error. This error is calculated through Eq. 8, relative percentage error. On the other hand, MSE is calculated from Eq. 9.

$$
\begin{gathered}
E R P=\frac{|y-\hat{y}|}{y} * 100 \\
M S E=\frac{1}{n} \sum_{i=1}^{n}\left(y_{i}-\widehat{y_{i}}\right)
\end{gathered}
$$

Where $y$ is the expected or ideal value and $\hat{y}$ is the estimated value.

As shown in Table 1, the percentage of the average relative error ERP Eq. 8 decreases when using the first proposed modification introduced in Sect. 4, which consists of verifying that the block with the minimum standard deviation $\left(\sigma_{\min 1}\right)$ is adequate. 


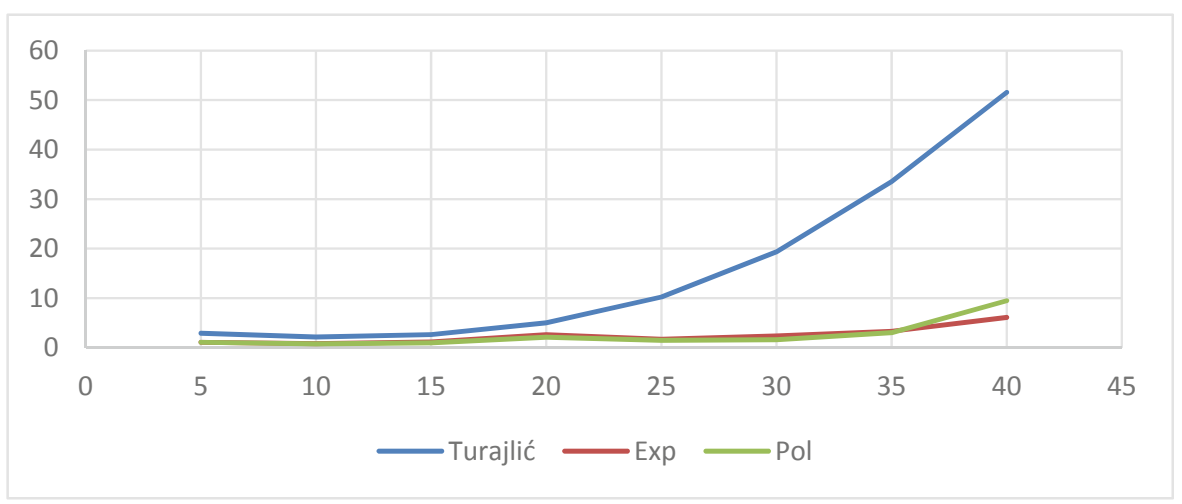

Fig. 4. MSE for each added noise level $\left(\sigma_{A D D}\right)$, using $11 \%$ of the image.

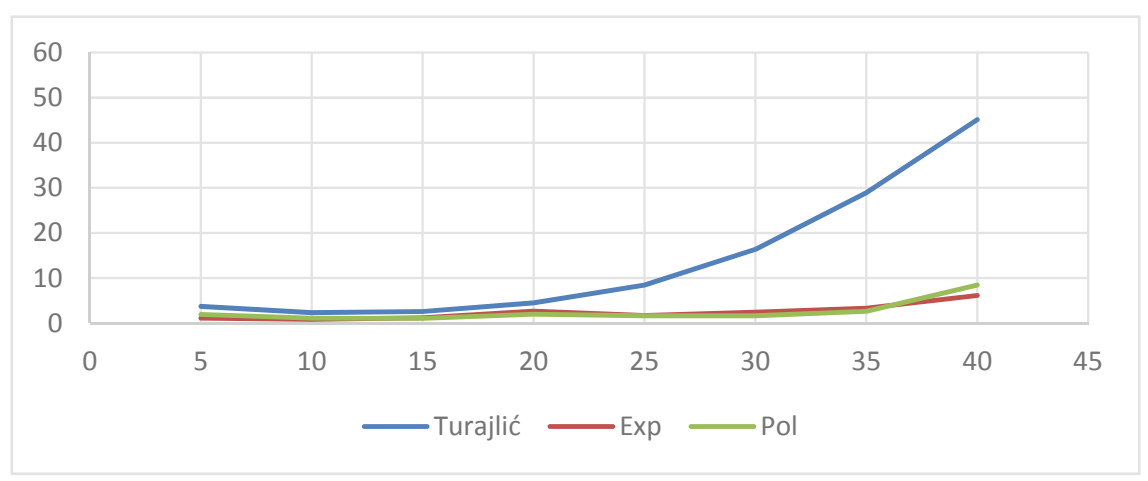

Fig. 5. MSE for each added noise level $\left(\sigma_{A D D}\right)$, using $15 \%$ of the image.

Table 1. Average ERP with the 40 test images between $\sigma_{\min 1}$ and the total noise standard deviation Eq. 5 of each image.

\begin{tabular}{l|l|r|l|l|l|l|l|l}
\hline Mode & 5 & 10 & 15 & \multicolumn{1}{l|}{20} & \multicolumn{1}{l}{25} & \multicolumn{1}{l}{30} & \multicolumn{1}{l}{35} & \multicolumn{1}{l}{40} \\
\hline Turajlić & $19 \%$ & $11 \%$ & $10 \%$ & $11 \%$ & $13 \%$ & $15 \%$ & $17 \%$ & $18 \%$ \\
\hline Proposed & $18 \%$ & $8 \%$ & $6 \%$ & $6 \%$ & $5 \%$ & $5 \%$ & $5 \%$ & $6 \%$ \\
\hline
\end{tabular}

Figure 6 shows the average method execution time for each added noise level. As expected, the proposed method run time is higher compared to the original due to the higher number of steps added. As can also be observed, the working area of the image does not significantly affect the execution time. 
As shown in Fig. 4 y 5, if the Turajlić method is used in both cases (with $11 \%$ and $15 \%$ of the image), the noise estimate is further away from the real value and it is observed that from an aggregated noise level of $15\left(\sigma_{A D D}=15\right)$, the mean square error increases exponentially. On the contrary, the proposed methods achieve a better estimate regardless of the amount of noise added to the image, obtaining similar results for the different added noise levels, except when the additional noise has a deviation of 40, where the exponential approximation (Exp) allows a better estimate. It should be noted that the error of noise estimation obtained with Turajlić, original method, found in this study, is much higher than that mentioned in the original paper [9]. This is probably due to the fact that the images used in this study are different from those used in the original paper, which are not available.
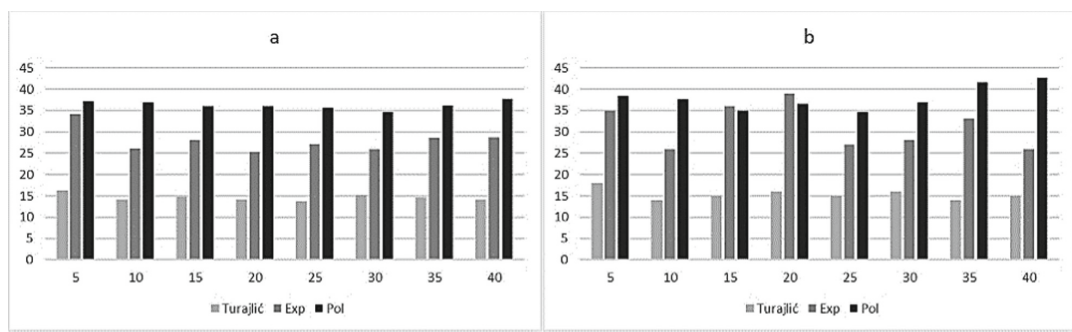

Fig. 6. Execution times in milliseconds. a) Using $11 \%$ of the image. b) Using $15 \%$ of the image.

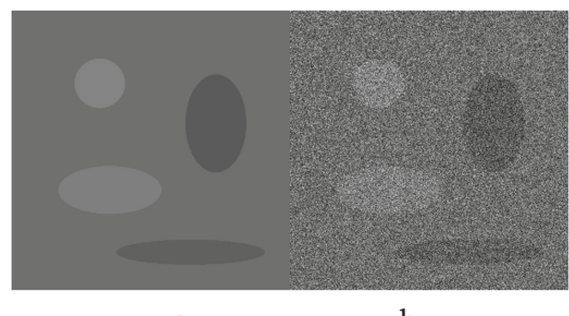

a

$\mathrm{b}$

Fig. 7. a) Original image. b) Images degraded by noise $\sigma_{A D D}=40$.

Table 2. Percentage of acceptable estimates using $11 \%$ and $15 \%$ of the image.

\begin{tabular}{l|l|l}
\hline Mode & $11 \%$ & $15 \%$ \\
\hline Turajlić & $37.81 \%$ & $37.19 \%$ \\
\hline Exp & $73.13 \%$ & $72.81 \%$ \\
\hline Pol & $71.88 \%$ & $69.69 \%$ \\
\hline
\end{tabular}


The modifications produced an increase of slightly less than twice the percentage of acceptable estimates from the original method (Table 2). It should be noted that during the process it was found that the improved method had a drawback since for images with a large amount of black or white background (with minimum or maximum pixel intensity) presented an appreciable estimation error greater than $30 \%$.

As can be seen in Figs. 4 and 5 the estimation error increases significantly when the degradation is greater than 35 , which is caused by the fact that the magnitude of changes due to noise are similar to those produced by the edges of the images, as illustrated in Fig. 7 and, therefore, the sub-block with minimum standard deviation may erroneously include edges of the image.

\section{Conclusions}

In this document an improvement was made on the original noise estimation method of Turajlić. The changes introduced allowed more accurate results to be obtained, improving the Gaussian noise level estimate by more than $30 \%$, regardless of the percentage of blocks, 11 and 15\%, taken in the first stage to estimate the noise in the second. In both cases the mean square error and the computation time is similar.

The first improvement, which consists of discarding the blocks whose Gaussian noise function is truncated, allows to decrease the noise estimation error. Furthermore, two proposed approaches, polynomial and exponential, for the calculation of an adjustment factor allowed to improve even more the accuracy of the estimation, being the error similar in both cases. However, when the noise level is higher than $\sigma=35$ the exponential approach gives better results. The estimation method fails when the noise level is very close to the amplitude of the borders of the objects in the image, since abrupt changes produced by noise are confused with those of the signal.

Acknowledgements. This work was supported by project \# 19-488-INT Universidad de Ibagué.

\section{Appendix}

Forty images, some of them shown in Fig. 8, were used in this study, Twenty-seven taken from [10], seven from [12] and four from [11]. The remaining two are synthetic images created by the image processing research laboratory Lún of the Universidad de Ibagué. 


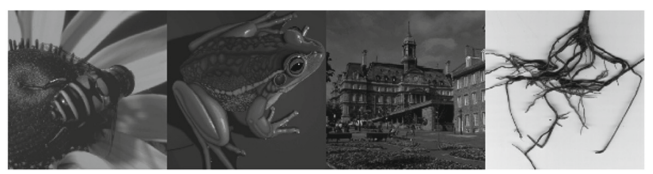

a.

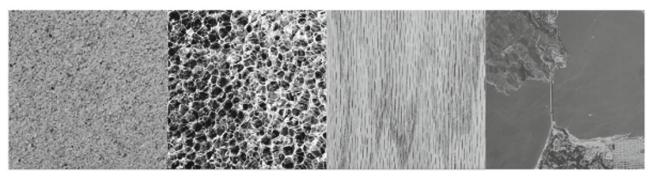

b.

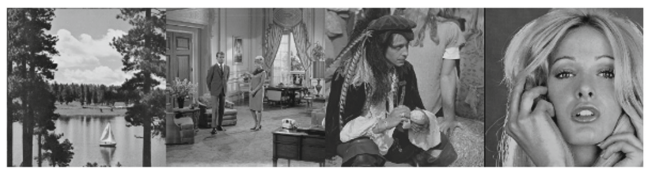

c.

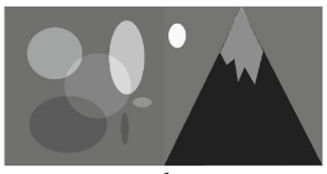

d.

Fig. 8. Images of size $512 \times 512$ pixels used in this study. a) Obtained from [10], b) from [12] and c) from [11].

\section{References}

1. Kovesi, P.: Image features from phase congruency. Videre J. Comput. Vis. Res. 1(3), 1-26 (1999)

2. Perona, P., Jitendra, M.: Scale-Space and Edge Detection Using Anisotropic Diffusion. IEEE, Berkeley (1990)

3. Aharon, M., Elad, M., Bruckstein, A.: K-SVD: an algorithm for designing overcomplete dictionaries for sparse representation. IEEE Trans. Signal Process. 54(11), 4311-4322 (2006)

4. Park, R.-H., Yang, S., Jung, J.-H., Shin, D.-H.: Block-based noise estimation using adaptive Gaussian filtering. IEEE Trans. Consum. Electron. 51(1), 218-226 (2005)

5. Begović, A., Turajlić, E.: Noise estimation using adaptive gaussian filtering and variable block size image segmentation. In: IEEE EUROCON 2017 - 17th International Conference on Smart Technologies, pp. 250-254 (2017)

6. Liu, W.: Additive white Gaussian noise level estimation based on block SVD. In: IEEE Workshop on Electronics, Computer and Applications, pp. 960-963 (2014)

7. Tang, C., Yang, X., Zhai, G.: Dual-transform based noise estimation. In: Proceedings of the IEEE International Conference on Multimedia Expo (ICME), pp. 991-996, July 2012

8. Forero, M.G., Miranda, S.L.: Gaussian noise estimation methods. In: Proceedings of the SPIE 11137, Applications of Digital Image Processing XLII, San Diego (2019)

9. Turajlić, E.: A fast noise level estimation algorithm based on adaptive image segmentation and Laplacian convolution. In: MIPRO, pp. 486-491 (2017)

10. Universidad de Granada: Test Images. http://decsai.ugr.es/cvg/dbimagenes/

11. ImageProcessing.com. http://www.imageprocessingplace.com/root_files_V3/image_databa ses.htm 
12. University of Southern California: The USC-SIPI Image Database. http://sipi.usc.edu/databa se/database.php

13. Rasband, W.S.: ImageJ, U. S. National Institutes of Health, Bethesda, Maryland, USA 19972018. https://imagej.nih.gov/ij/

14. Immerkær, J.: Fast noise variance estimation. Comput. Vis. Image Underst. 64, 300-301 (1996) 